PROCEEDINGS OF THE

AMERICAN MATHEMATICAL SOCIETY

Volume 133, Number 7, Pages 1873-1879

S 0002-9939(05)07903-7

Article electronically published on February 24, 2005

\title{
ON IDENTITIES IN GROUPS OF FRACTIONS OF CANCELLATIVE SEMIGROUPS
}

\author{
S. V. IVANOV AND A. M. STOROZHEV
}

(Communicated by Jonathan I. Hall)

\begin{abstract}
To solve two problems of Bergman stated in 1981, we construct a group $G$ such that $G$ contains a free noncyclic subgroup (hence, $G$ satisfies no group identity) and $G$, as a group, is generated by its subsemigroup that satisfies a nontrivial semigroup identity.
\end{abstract}

\section{INTRODUCTION}

A semigroup $S$ is called cancellative if for arbitrary $a, b, x \in S$ either of equalities $x a=x b, a x=b x$ implies that $a=b$.

A semigroup $S$ is said to satisfy the left (resp. right) Ore condition if for arbitrary $a, b \in S$ there are $x, y \in S$ such that $x a=y b$ (resp. $a x=b y$ ).

It is well known that a cancellative semigroup $S$ with the left (or right) Ore condition embeds in a group $\mathcal{F}(S)=S^{-1} S=S S^{-1}$ of its fractions (see M53, [NT63], CP67, Theorem 1.23]; recall that, in general, a cancellative semigroup need not embed in a group, see [M37]) and a group $\mathcal{F}(S)$ of fractions of $S$ is unique in the sense that if $G$ is a group such that $G$ contains $S$ and $G$, as a group, is generated by $S$, then $G$ is naturally isomorphic to $\mathcal{F}(S)$. In particular, if $S$ is a cancellative semigroup satisfying a nontrivial identity, then $S$ satisfies (both) Ore conditions and so $S$ embeds in the group $\mathcal{F}(S)$ of its fractions.

If $S$ is an abelian cancellative semigroup, then the group of its fractions is also abelian. Following Mal'tsev [M53] (see also [NT63]), a semigroup is called nilpotent of class $n$ if $S$ satisfies a semigroup identity of the form $X_{n} \equiv Y_{n}$, where $X_{n}, Y_{n}$ are words in letters $x, y, u_{1}, \ldots, u_{n}$, defined inductively by $X_{0}=x, Y_{0}=y$ and, for $n \geq 0$, by $X_{n+1}=X_{n} u_{n+1} Y_{n}, Y_{n+1}=Y_{n} u_{n+1} X_{n}$. In [M53], Mal'tsev proved that if $S$ is a nilpotent semigroup of class $n$, then $\mathcal{F}(S)$ is a nilpotent group of class $n$. It was also shown in [M53] that a group $K$ is nilpotent of class $n$ (in the sense of group theory) if and only if the identity $X_{n} \equiv Y_{n}$ holds in $K$.

In connection with the results stated above, Bergman [B81] posed the following natural problem (which, in a different form, is also mentioned by Shevrin and Suhanov in [SS89, Question 11.1]): Suppose a cancellative semigroup $S$ satisfies a nontrivial semigroup identity. Does then the group $\mathcal{F}(S)$ of fractions of $S$ satisfy all the semigroup identities that hold in $S$ ? Bergman [B81] also proposed a weaker

Received by the editors November 2, 2003.

2000 Mathematics Subject Classification. Primary 20E10, 20F05, 20F06, 20M05.

The first author was supported in part by NSF grants DMS 00-99612, 04-00746.

(C)2005 American Mathematical Society Reverts to public domain 28 years from publication 
problem: If a cancellative semigroup $S$ satisfies a nontrivial semigroup identity, must then the group $\mathcal{F}(S)$ of its fractions satisfy a nontrivial group identity?

Krempa and Macedońska KM92 proved that if an identity of the form $x^{n} y^{n} \equiv$ $y^{n} x^{n}, n \geq 1$, holds in a cancellative semigroup $S$, then every semigroup identity of $S$ holds in $\mathcal{F}(S)$ (hence, both of Bergman's problems have positive solutions for the identity $\left.x^{n} y^{n} \equiv y^{n} x^{n}\right)$. Krempa and Macedońska [KM92] also observed that the same result holds for the Mal'tsev nilpotent identity $X_{n} \equiv Y_{n}, n \geq 1$.

In this paper, we construct an $m$-generator group $\mathcal{G}=\left\langle\bar{a}_{1}, \ldots, \bar{a}_{m}\right\rangle, m>1$, generated by $\bar{a}_{1}, \ldots, \bar{a}_{m}$, such that $\mathcal{G}$ contains a free noncyclic subgroup (hence, no group identity holds in $\mathcal{G}$ ) and the subsemigroup $\mathcal{S}=\left\langle\bar{a}_{1}, \ldots, \bar{a}_{m}\right\rangle^{+}$of $\mathcal{G}$, generated by $\bar{a}_{1}, \ldots, \bar{a}_{m}$, satisfies a nontrivial semigroup identity (namely, $w_{L}(x, y) \equiv$ $w_{R}(x, y)$; see (1)-(2) below). Since $\mathcal{S}$ is obviously cancellative and $\mathcal{G}$, as a group, is generated by $\mathcal{S}$, it follows that both of Bergman's problems are solved in the negative.

It should be pointed out that, in the 1980s, Rips suggested a similar approach to solve Bergman's problems in the negative. However, to the best of our knowledge, no details of his construction are available.

Now we will give details of our construction. Consider the following positive words:

$$
w_{L}(x, y)=\left(x^{d} y^{d}\right)^{n+1^{2}} x\left(x^{d} y^{d}\right)^{n+2^{2}} x \ldots\left(x^{d} y^{d}\right)^{n+\left(h_{0}-1\right)^{2}} x\left(x^{d} y^{d}\right)^{n+h_{0}^{2}} x
$$

and

$$
\begin{gathered}
w_{R}(x, y)=x\left(x^{d} y^{d}\right)^{n-h_{0}^{2}\left(2 h_{0}-3\right)} x\left(x^{d} y^{d}\right)^{n+\left(2 h_{0}-1\right)^{2}} x\left(x^{d} y^{d}\right)^{n+\left(2 h_{0}-2\right)^{2}} \ldots \\
\ldots x\left(x^{d} y^{d}\right)^{n+\left(h_{0}+2\right)^{2}} x\left(x^{d} y^{d}\right)^{n+\left(h_{0}+1\right)^{2}},
\end{gathered}
$$

where $h_{0}=h / 2, d, n$ are sufficiently large positive integers (with $n \gg d \gg h \gg 1$ ). Observe that it follows from the equality $1^{2}+2^{2}+\cdots+k^{2}=\frac{k(2 k+1)(k+1)}{6}$ that the sum of exponents on $x$ (resp. $y$ ) in $w_{L}(x, y)$ is equal to that on $x$ (resp. $y$ ) in $w_{R}(x, y)$. Note that the word

$$
w(x, y)=w_{L}(x, y) w_{R}(x, y)^{-1}
$$

was introduced in [OS96] to construct the first example of a finitely generated group $G_{0}$ such that $G_{0}$ satisfies a nontrivial semigroup identity (namely, $w_{L}(x, y) \equiv$ $\left.w_{R}(x, y)\right)$ and $G_{0}$ is not a periodic extension of a locally nilpotent group. However, the parameter $h$, used in the construction of $w(x, y)$ in OS96, should actually read $h / 2$ and so we put $h_{0}=h / 2$ in (11), (2) (recall that, according to the condition $R$ of [O89. Sect. 25], every 2-cell of second type must have exactly $h$ long sections).

We also recall that the question on the existence of a finitely generated group that satisfies a nontrivial semigroup identity and is not a periodic extension of a locally nilpotent group was asked by Longobardi, Maj and Rhemtulla LMR95] and goes back to a result of Rosenblatt [R74] (rediscovered in [LMR95]) that a finitely generated solvable group which contains no free subsemigroups of rank 2 is nilpotent-by-finite. Here is our main result.

Theorem. There exists an m-generator torsion-free group $\mathcal{G}=\left\langle\bar{a}_{1}, \ldots, \bar{a}_{m}\right\rangle, m \geq$ 2 , such that $\mathcal{G}$ contains a subgroup isomorphic to a free group of rank 2 (e.g., generated by $\bar{a}_{1} \bar{a}_{2}^{-1} \bar{a}_{1}$ and $\left.\bar{a}_{2} \bar{a}_{1}^{-1} \bar{a}_{2}\right)$ and the nontrivial semigroup identity $w_{L}(x, y) \equiv$ $w_{R}(x, y)\left(\right.$ see (1) -(2)) holds in the subsemigroup $\mathcal{S}=\left\langle\bar{a}_{1}, \ldots, \bar{a}_{m}\right\rangle^{+}$of $\mathcal{G}$, generated by $\bar{a}_{1}, \ldots, \bar{a}_{m}$. 


\section{InduCtive CONSTRUCTION}

To prove this Theorem, we will inductively construct a presentation for the group $\mathcal{G}$ by means of generators and defining relations and use the geometric machinery of graded diagrams, developed by Ol'shanskii [085], O89] and refined by the authors in [IS03, to study this group. In particular, we will use the notation and terminology of [089, and all notions that are not defined in this paper can be found in O89.

As in [089], we will use numerical parameters

$$
\alpha \succ \beta \succ \gamma \succ \delta \succ \varepsilon \succ \zeta \succ \eta \succ \iota
$$

and $h=\delta^{-1}, d=\eta^{-1}, n=\iota^{-1}(h, d, n$ were already used in (1)-(2) $)$ and employ the least parameter principle (LPP) (according to LPP a small positive value for, say, $\zeta$ is chosen to satisfy all inequalities whose smallest (in terms of the relation $\succ)$ parameter is $\zeta$ ).

Let $\mathcal{A}=\left\{a_{1}, \ldots, a_{m}\right\}$ be an alphabet, $m>1$, and let $F(\mathcal{A})$ be the free group in $\mathcal{A}$. Elements of $F(\mathcal{A})$ are referred to as words in $\mathcal{A}^{ \pm 1}=\mathcal{A} \cup \mathcal{A}^{-1}$ or just words. We will say that $U \in F(\mathcal{A})$ is a positive word if $U$ has no occurrences of $a_{1}^{-1}, \ldots, a_{m}^{-1}$. A word $V$ will be called regular if at least one of $V, V^{-1}$ contains a positive subword of length 3 .

Denote $G(0)=F(\mathcal{A})$ and let the set $\mathcal{R}_{0}$ of defining words of rank 0 be empty. To define the group $G(i)$ by induction on $i \geq 1$, assume that the group $G(i-1)$ is already constructed by its presentation

$$
G(i-1)=\left\langle\mathcal{A} \| R=1, R \in \mathcal{R}_{i-1}\right\rangle .
$$

Let $X_{i}$ be a set of words (in $\mathcal{A}^{ \pm 1}$ ) of length $i$, called periods of rank $i$, which is maximal with respect to the following two properties:

(A1) If $A \in X_{i}$, then $A$ (that is, the image of $A$ in $G(i-1)$ ) is not conjugate in $G(i-1)$ to a power of a word of length $<|A|=i$.

(A2) If $A, B$ are distinct elements of $X_{i}$, then $A$ is not conjugate in $G(i-1)$ to $B$ or $B^{-1}$.

If the images of two words $X, Y$ are equal in the group $G(i-1), i \geq 1$, then we will say that $X$ is equal in rank $i-1$ to $Y$ and write $X \stackrel{i-1}{=} Y$. Analogously, we will say that two words $X, Y$ are conjugate in rank $i-1$ if their images are conjugate in the group $G(i-1)$. As in [089], a word $A$ is called simple in rank $i-1, i \geq 1$, if $A$ is conjugate in rank $i-1$ neither to a power $B^{\ell}$, where $|B|<|A|$ nor to a power of period of some rank $\leq i-1$. We will also say that two pairs $\left(X_{1}, X_{2}\right)$, $\left(Y_{1}, Y_{2}\right)$ of words are conjugate in rank $i-1, i \geq 1$, if there is a word $W$ such that $X_{1} \stackrel{i-1}{=} W Y_{1} W^{-1}$ and $X_{2} \stackrel{i-1}{=} W Y_{2} W^{-1}$.

Consider the set of all possible pairs $(X, Y)$ of words in $\mathcal{A}^{ \pm 1}$. This set is partitioned by equivalence classes $\mathcal{C}_{\ell}, \ell=1,2, \ldots$, of the equivalence relation $\sim$ defined by $\left(X_{1}, Y_{1}\right) \sim\left(X_{2}, Y_{2}\right)$ if and only if the pairs $\left(X_{1}^{d} Y_{1}^{d}, w\left(X_{1}, Y_{1}\right)\right)$ and $\left(X_{2}^{d} Y_{2}^{d}, w\left(X_{2}, Y_{2}\right)\right)$, where $w(x, y)$ is given by (3), are conjugate in rank $i-1$. It is convenient to enumerate (in some way)

$$
\mathcal{C}_{A^{f}, 1}, \mathcal{C}_{A^{f}, 2}, \ldots
$$

all classes of pairs $(X, Y)$ such that $w(X, Y) \stackrel{i-1}{\neq} 1$ and $X^{d} Y^{d}$ is conjugate in rank $i-1$ to some power $A^{f}$, where $A \in X_{i}$ and $f$ are fixed. 
It follows from definitions that every class $\mathcal{C}_{A^{f}, j}$ contains a pair

$$
\left(X_{A^{f}, j}, \bar{Y}_{A^{f}, j}\right)
$$

with the following properties. The word $X_{A^{f}, j}$ is graphically (that is, letter-byletter) equal to a power of $B_{A^{f}, j}$, where $B_{A^{f}, j}$ is simple in rank $i-1$ or a period of rank $\leq i-1 ; \bar{Y}_{A^{f}, j} \equiv Z_{A^{f}, j} Y_{A^{f}, j} Z_{A^{f}, j}^{-1}$, where the symbol "三" means the graphical equality, $Y_{A^{f}, j}$ is graphically equal to a power of $C_{A^{f}, j}$, where $C_{A^{f}, j}$ is simple in rank $i-1$ or a period of rank $\leq i-1$. We can also assume that if $D_{1} \in\left\{A, B_{A^{f}, j}, C_{A^{f}, j}\right\}$ is conjugate in rank $i-1$ to $D_{2}^{ \pm 1}$, where $D_{2} \in\left\{A, B_{A^{f}, j}, C_{A^{f}, j}\right\}$, then $D_{1} \equiv D_{2}$. Finally, the word $Z_{A^{f}, j}$ is picked for fixed $X_{A^{f}, j}, Y_{A^{f}, j}$ so that the length $\left|Z_{A^{f}, j}\right|$ is minimal (and the pair $\left(X_{A^{f}, j}, Z_{A^{f}, j} Y_{A^{f}, j} Z_{A^{f}, j}^{-1}\right)$ belongs to $\left.\mathcal{C}_{A^{f}, j}\right)$. Similar to 085 , O89, IS03, the triple $\left(X_{A^{f}, j}, Y_{A^{f}, j}, Z_{A^{f}, j}\right)$ is called an $\left(A^{f}, j\right)$-triple corresponding to the class $\mathrm{C}_{A^{f}, j}($ in $\operatorname{rank} i-1)$.

Now for every class $\mathcal{C}_{A^{f}, j}$, where $A$ is a regular word, we pick a corresponding $\left(A^{f}, j\right)$-triple

$$
\left(X_{A^{f}, j}, Y_{A^{f}, j}, Z_{A^{f}, j}\right)
$$

in rank $i-1$ and construct a defining word $R_{A^{f}, j}$ of rank $i$ as follows. Pick a word $W_{A^{f}, j}$ of minimal length so that

$$
X_{A^{f}, j}^{d} \bar{Y}_{A^{f}, j}^{d} \stackrel{i-1}{=} W_{A^{f}, j} A^{f} W_{A^{f}, j}^{-1} .
$$

Let $T_{A^{f}, j}$ be a word of minimal length such that

$$
T_{A^{f}, j} \stackrel{i=1}{=} W_{A^{f}, j}^{-1} X_{A^{f}, j} W_{A^{f}, j} .
$$

According to (3) (see also (11)-(2)), we set

$$
\begin{array}{r}
R_{A^{f}, j}=A^{\left(n+1^{2}\right) f} T_{A^{f}, j} A^{\left(n+2^{2}\right) f} T_{A^{f}, j} \ldots A^{\left(n+\left(h_{0}-1\right)^{2}\right) f} T_{A^{f}, j} A^{\left(n+h_{0}^{2}\right) f} T_{A^{f}, j} \\
A^{\left(-n-\left(h_{0}+1\right)^{2}\right) f} T_{A^{f}, j}^{-1} A^{\left(-n-\left(h_{0}+2\right)^{2}\right) f} T_{A^{f}, j}^{-1} \ldots A^{\left(-n-\left(2 h_{0}-2\right)^{2}\right) f} T_{A^{f}, j}^{-1} \\
A^{\left(-n-\left(2 h_{0}-1\right)^{2}\right) f} T_{A^{f}, j}^{-1} A^{\left(-n+h_{0}^{2}\left(2 h_{0}-3\right)\right) f} T_{A^{f}, j}^{-1} .
\end{array}
$$

It follows from definitions that the word $R_{A^{f}, j}$ is conjugate in rank $i-1$ (by $W_{A^{f}, j}^{-1}$ ) to the word $w\left(X_{A^{f}, j}, \bar{Y}_{A^{f}, j}\right) \stackrel{i-1}{\neq} 1$.

The set $\mathcal{S}_{i}$ of defining words of rank $i$ consists of all possible words $R_{A^{f}, j}$ given by (41) (over all equivalence classes $\mathcal{C}_{A^{f}, j}$, where $A \in \mathcal{X}_{i}$ is a regular word). Finally, we put $\mathcal{R}_{i}=\mathcal{R}_{i-1} \cup \mathcal{S}_{i}$ and set

$$
G(i)=\left\langle\mathcal{A} \| R=1, R \in \mathcal{R}_{i}\right\rangle .
$$

The inductive definition of groups $G(i), i \geq 0$, is now complete and we can consider the limit group $\mathcal{G}$ given by defining words of all ranks $j=1,2, \ldots$.

$$
\mathcal{G}=G(\infty)=\left\langle\mathcal{A} \| R=1, R \in \bigcup_{j=0}^{\infty} \mathcal{R}_{j}\right\rangle .
$$




\section{Proof of the Theorem}

First we will establish several lemmas.

Lemma 1. The presentation (5) of $G(i)$ satisfies the condition $R$ of $[089$, Sect. 25].

Proof. Recall that in article [OS96] the free group $F(\mathcal{A}) / w(F(\mathcal{A}))$ of the variety of groups, defined by the identity $w(x, y) \equiv 1$ (see (3i)), is constructed. The inductive construction of a presentation for the group $F(\mathcal{A}) / w(F(\mathcal{A}))$ in [OS96] is analogous to our construction of the group $\mathcal{G}=G(\infty)$ (the only difference is that $A \in X_{i}$ is arbitrary in [S96] and now we require that $A$ be regular) and many technical results of [OS96] are reproved in our situation without any changes. In particular, repeating the arguments of the proofs of Lemmas $4-6$ in OS96], we can prove Lemma 1]

Now suppose that $X, Y$ are some words such that $[X, Y]=X Y X^{-1} Y^{-1} \stackrel{i}{\neq} 1$. Conjugating the pair $(X, Y)$ in rank $i$, if necessary, we can assume that $X \equiv B^{f_{B}}$, $Y \equiv Z C^{f_{C}} Z^{-1}$, where each of $B, C$ is either simple in rank $i$ or a period of some rank $\leq i$ and, when $B^{f_{B}}, C^{f_{C}}$ are fixed, the word $Z$ is picked to have minimal length. We also consider the following equality:

$$
X^{d} Y^{d} \stackrel{i}{=} W_{A} A^{f_{A}} W_{A}^{-1},
$$

where $A$ is either simple in rank $i$ or a period of some rank $\leq i$ and the conjugating word $W_{A}$ is picked (when $A$ is fixed) to have minimal length. Without loss of generality, we can also assume that if $D_{1} \in\{A, B, C\}$ is conjugate in rank $i$ to $D_{2}^{ \pm 1}$, where $D_{2} \in\{A, B, C\}$, then $D_{1} \equiv D_{2}$.

Lemma 2. In the foregoing notation, the following inequalities hold:

$$
0<\left|f_{A}\right| \leq 100 \zeta^{-1}, \quad \max \left(\left|B^{d f_{B}}\right|,\left|C^{d f_{C}}\right|\right) \leq \zeta^{-2}\left|A^{f_{A}}\right|, \quad|Z|<3 \zeta^{-2}\left|A^{f_{A}}\right| .
$$

Proof. These inequalities are proved analogously to inequalities (13)-(14) of Lemma 6 [S03] (see also Lemma 3 in [S94]).

Lemma 3. Let $\widetilde{X}$ and $\widetilde{Y}$ be positive words. Then $w(\widetilde{X}, \widetilde{Y})=1$ in the group $G(\infty)$ given by presentation (6).

Proof. Arguing on the contrary, assume that

$$
w(\widetilde{X}, \widetilde{Y}) \neq 1
$$

in $G(\infty)$. Let $A$ be a period of some rank such that $A^{f_{A}}$ for some $f_{A}$ is conjugate in $G(\infty)$ to $\widetilde{X}^{d} \widetilde{Y}^{d}$. (The existence of such an $A$ follows from definitions; see also Lemma 18.1 in $[089]$.) Note that, in view of (7), $[\widetilde{X}, \widetilde{Y}] \neq 1$ in $G(\infty)$. Hence, by Lemma 2 we can replace the pair $(\widetilde{X}, \widetilde{Y})$ by a conjugate in the group $G(\infty)$ pair $(X, Y)$ such that $X \equiv B^{f_{B}}, Y \equiv Z C^{f_{C}} Z^{-1}$, and $X^{d} Y^{d}=W_{A} A^{f_{A}} W_{A}^{-1}$ in $G(\infty)$, where $B, C$ are some periods and the inequalities of Lemma 2 hold. (Note that $X, Y$ need not be positive words.) In particular,

$$
\left|X^{d} Y^{d}\right| \leq\left|B^{d f_{B}}\right|+\left|C^{d f_{C}}\right|+2|Z|<8 \zeta^{-2}\left|A^{f_{A}}\right| .
$$

Let $\widetilde{\Delta}$ be a reduced annular diagram of $\operatorname{rank} \widetilde{i}$ for conjugacy of $\widetilde{X}^{d} \widetilde{Y}^{d}$ and $A^{f_{A}}$. By Theorem 22.4 in [089] applied to $\Delta$, we have

$$
(1-\beta)\left|A^{f_{A}}\right| \leq\left|\widetilde{X}^{d} \widetilde{Y}^{d}\right| \text {. }
$$


It follows from the definition of the word $w(x, y)$ (see (3)) that the sums of exponents on $x$ and $y$ in $w(x, y)$ are 0 . Since all defining words of presentation (6) are values of $w(x, y)$, these defining words are in the commutator subgroup of $F(\mathcal{A})$. Hence, the total sum $\sigma\left(\widetilde{X}^{d} \widetilde{Y}^{d}\right)+\sigma\left(A^{-f_{A}}\right)$ of exponents on letters $a_{1}, \ldots, a_{m}$ in words $\widetilde{X}^{d} \widetilde{Y}^{d}$ and $A^{-f_{A}}$ is equal to 0 . Let $\sigma^{+}\left(A^{f_{A}}\right)$ denote the sum of all positive exponents on letters $a_{1}, \ldots, a_{m}$ in $A^{f_{A}}$. Since $\widetilde{X}^{d} \widetilde{Y}^{d}$ is conjugate in $G(\infty)$ to $A^{f_{A}}$ and $\widetilde{X}^{d}, \widetilde{Y}^{d}$ are positive words, it follows that

$$
\sigma^{+}\left(A^{f_{A}}\right) \geq\left|\widetilde{X}^{d} \widetilde{Y}^{d}\right| .
$$

In view of inequalities (19)-(10), we further have

$$
\sigma^{+}\left(A^{f_{A}}\right) \geq(1-\beta)\left|A^{f_{A}}\right| .
$$

Assume that $A$ is not regular. Then the sum of the positive exponents on $a_{1}, \ldots, a_{m}$ in $A^{f_{A} /\left|f_{A}\right|}$ is at most $\frac{2}{3}(|A|+1)$. Therefore, $\sigma^{+}\left(A^{f_{A}}\right) \leq \frac{2}{3}\left|f_{A}\right|(|A|+1)$ and, in view of (11), we get $\frac{2}{3}(|A|+1) \geq(1-\beta)|A|$, which implies that $|A|<3$ (LPP). On the other hand, it follows from Lemma 2 that

$$
|A| \geq\left|f_{A}\right|^{-1} \zeta^{2}\left|X^{d}\right| \geq 100^{-1} \zeta^{3} d>3
$$

(LPP: $\zeta \succ \eta=d^{-1}$ ). This contradiction to $|A|<3$ shows that $A$ is regular.

Now we consider a reduced annular diagram $\Delta$ of some rank $i^{\prime}$ for conjugacy of $X^{d} Y^{d}$ and $A^{f_{A}}$. By Lemma 1 and by Lemma 22.1 in [089], $\Delta$ can be cut into a simply connected diagram $\Delta_{1}$ along a simple path $t$ which connects points on distinct components of $\partial \Delta$ with $|t|<\gamma|\partial \Delta|$. Therefore, in view of estimate (8),

$$
\begin{aligned}
\left|\partial \Delta_{1}\right|<(1+2 \gamma)|\partial \Delta|=(1+ & 2 \gamma)\left(\left|X^{d} Y^{d}\right|+\left|A^{f_{A}}\right|\right) \\
& <(1+2 \gamma)\left(8 \zeta^{-2}+1\right)\left|A^{f_{A}}\right|<10^{3} \zeta^{-3}|A|<\frac{1}{2} n|A|
\end{aligned}
$$

(LPP: $\gamma \succ \zeta \succ \iota=n^{-1}$ ). Then, by Lemma 1 and by Lemmas 20.4 and 23.16 in (O89] applied to $\Delta_{1}$, the diagram $\Delta_{1}$ contains no 2-cells of rank $\geq|A|$, whence $\Delta_{1}$, $\Delta$ are diagrams of rank $|A|-1$. Since $A \in \mathcal{X}_{|A|}$ is a regular word, it follows from the construction of defining words of rank $|A|$ that there will be a defining word in $\mathcal{S}_{|A|}$ which guarantees that $w(X, Y) \stackrel{|A|}{=} 1$. Consequently, $w(\tilde{X}, \tilde{Y})=1$ in $G(\infty)$ and a contradiction to assumption (7) proves Lemma 3 ,

Proof of the Theorem. It follows from definitions, Lemma 1 and Lemma 25.2 in 089 that the group $\mathcal{G}=G(\infty)=\left\langle a_{1}, \ldots, a_{m}\right\rangle$, given by presentation (6), is torsion-free. By Lemma 3 the subsemigroup $\mathcal{S}=\left\langle a_{1}, \ldots, a_{m}\right\rangle^{+}$of $\mathcal{G}$, generated by $a_{1}, \ldots, a_{m}$, satisfies the nontrivial semigroup identity $w_{L}(x, y) \equiv w_{R}(x, y)$. It remains to show that $\mathcal{G}$ contains a subgroup isomorphic to a free group of rank 2 . To do this, consider words $V_{1}=a_{1} a_{2}^{-1} a_{1}$ and $V_{2}=a_{2} a_{1}^{-1} a_{2}$. Assume that there is a nonempty cyclically reduced word $U\left(V_{1}, V_{2}\right)$ in $V_{1}^{ \pm 1}, V_{2}^{ \pm 1}$ such that $U\left(V_{1}, V_{2}\right)=1$ in the group $\mathcal{G}=G(\infty)$. Then we can consider a reduced disk diagram $\Delta$ of positive rank, the label of whose boundary $\partial \Delta$ is $U\left(V_{1}, V_{2}\right)$. It follows from the definition of defining words of $\mathcal{G}=G(\infty)$ and Theorem 22.2 in 089 applied to $\Delta$ that $U\left(V_{1}, V_{2}\right)$ is a regular word. This, however, is obviously false. This contradiction shows that the subgroup $\left\langle V_{1}, V_{2}\right\rangle$ of $\mathcal{G}$ is free of rank 2 and the Theorem is proved. 


\section{ACKNOWLEDGEMENTS}

The authors wish to thank George Bergman for pointing out a typo in the word (2) and for other useful comments.

\section{REFERENCES}

[B81] G.M. Bergman, Hyperidentities of groups and semigroups, Aequat. Math. 23(1981), 55-65. MR0667217 (83m:08016)

[CP67] A.H. Clifford and G.B. Preston, The algebraic theory of semigroups, AMS Math. Surveys, vol. 7, 1967. MR0218472 (36:1558)

[IS03] S.V. Ivanov and A.M. Storozhev, Non-hopfian relatively free groups, submitted, preprint available at http://arXiv.org.

[KM92] J. Krempa and O. Macedońska, On identites of cancellative semigroups, Contemp. Math. 131(1992), 125-134. MR1175878 (93g:20108)

[LMR95] P. Longobardi, M. Maj and A.H. Rhemtulla, Groups with no free subsemigroups, Trans. Amer. Math. Soc. 347(1995), 1419-1427. MR1277124 (95g:20043)

[M37] A.I. Mal'tsev, On the immersion of an algebraic ring into a field, Math. Ann. 113(1937), 686-691.

[M53] A.I. Mal'tsev, Nilpotent semigroups, Uchen. Zap. Ivanovsk. Ped. Inst. 4(1953), 107-111. MF 0075959 (17:825d)

[NT63] B.H. Neumann and T. Taylor, Semigroups of nilpotent groups, Proc. Royal Soc. A 274(1963), 1-4. MR0159884 (28:3100)

[O85] A.Yu. Ol'shanskii, Varieties in which all finite groups are abelian, Mat. Sbornik 126(1985), 59-82. MR0773429 (86d:20031)

[O89] A.Yu. Ol'shanskii, Geometry of defining relations in groups, Nauka, Moscow, 1989; English translation in Math. and Its Appl., Soviet series 70, Kluwer Acad. Publ., 1991. MF,1191619 (93g:20071)

[OS96] A.Yu. Ol'shanskii and A.M. Storozhev, A group variety defined by a semigroup law, J. Austral. Math. Soc. (Series A) 60(1996), 255-259. MF1375590 (97b:20033)

[R74] J.M. Rosenblatt, Invariant measures and growth conditions, Trans. Amer. Math. Soc. 193(1974), 33-53. MR0342955 (49:7699)

[SS89] L.N. Shevrin and E.V. Suhanov, Structural aspects in the theory of semigroup varieties, Izv. Vuzov, Matematika 6(1989), 3-39. MF1017775 (91a:20071)

[S94] A.M. Storozhev, On abelian subgroups of relatively free groups, Comm. Algebra 22(1994), 2677-2701. MR1271632 (95d:20066)

Department of Mathematics, University of Illinois, Urbana, Illinois 61801

E-mail address: ivanov@math.uiuc.edu

Australian Mathematics Trust, University of Canberra, Belconnen, ACT 2616, AusTRALIA

E-mail address: andreis@amt.canberra.edu.au 